to understand the role of specific microRNAs as biomarkers for disease activity (DA) and progression. Our aim was to evaluate the peripheral blood (PB) expression of miR-155 in SLE patients and to determine its correlation with the DA in the clinical practice.

Materials and methods We studied 40 SLE patients and 32 healthy controls. miR-155 expression levels in whole PB samples were determined by PCR (SYBR Green technology). 2- $\delta \delta$ Ct method was used for analysis. The DA was assessed by SLE DA index (SLEDAI).

Results miR-155 was upregulated in $50.0 \%$ of the patients and without difference in its expression levels in 17 (42.5\%) of the patients. ROC curve analysis was conducted in order to evaluate the diagnostic accuracy of the $\mathrm{PB}$ expression levels of the studied miRNA. AUC for miR-155 was 0.691 (95\% CI: 0.566 to 0.817$), p=0.005$ with $77.5 \%$ sensitivity and $50.0 \%$ specificity when the RQ cut value was 1.03 . Levels of miR-155 correlated with the diagnosis (rs 0,330, p=0,005), with patient's age ( $r s$ 0,366, p=0,002) as well as with the presence of secondary Raynaud phenomenon (rs 0,250, $\mathrm{p}=0,035)$. There was no correlations with SLEDAI $(\mathrm{p}=0$, 894) nor with the immunological activity according to ANA titer $(p=0.399), \quad a-d s D N A \quad(p=0.817), \quad a-S m \quad(p=0.285), \quad a-$ b2GPI $(p=0.903)$, a-CL antibodies $(p=0.857)$ and C3 and C4 complement levels $(\mathrm{p}=0.062$ and $\mathrm{p}=0.550$, respectively).

Conclusions We found a dysregulation of miR-155 in SLE which could suggests its role in the disease pathogenesis. There was no correlation between PB levels of miR-155 and $\mathrm{DA}$ as a whole as well as with the immunological activity which might reflect the variants of SLE DA in the studied patients, the difference in their genetic background or in the used medications but larger study is needed to confirm these results in the clinical practice.

Acknowledgement The study was supported by Grant 53/2014 funded by Medical University Sofia, Bulgaria.

\section{PS2:42 UNMETHYLATED CPG-RICH DNA FRAGMENTS ARE ASSOCIATED WITH THE PRESENCE OF LUPUS NEPHRITIS AND INFLUENCE TLR9-MEDIATED RENAL RESPONSE}

P Korsten, D Tampe, GA Müller, M Zeisberg, B Tampe. University Medical Centre Göttingen, Department of Nephrology and Rheumatology, Göttingen, Germany

\subsection{6/lupus-2018-abstract.90}

Purpose In systemic lupus erythematosus (SLE), lupus nephritis (LN) represents one of the most severe organ complications. $\mathrm{LN}$ is associated with persistent inflammation and perpetuated fibroblast activation, both determined by epigenetic mechanisms involving aberrant $\mathrm{CpG}$ DNA promoter methylation. During SLE progression, global methylation patterns are commonly lost. CpG DNA promoter methylation patterns are not limited to the kidney, circulating CpG-rich DNA is also detectable in the blood. However, little is known about its specific contribution to determining disease progression. In the kidney, CpG-rich DNA activates TLR9 signalling mechanisms involved in inflammation and fibrogenesis. Based on these observations, we hypothesised that CpG-rich DNA promoter fragments potentially accelerate renal inflammation and fibrogenesis in SLE-associated LN.

Methods First, CPG-rich DNA from blood samples of SLE patients with and without LN were collected. Then, we tested how these DNA promoter fragments influenced the LN phenotype in a TMPD ('pristane')-induced mouse model. The renal response to the administration of either human or synthetic methylated/unmethylated CpG-rich DNA oligodinucleotides (ODN) was observed. Downstream effects of the administration of circuclating CpG-rich DNA fragments on TLR9-signalling were analysed in endothelial cell cultures.

Results Circulating CpG-rich DNA promoter fragments are detectable in SLE patients" blood. LN was associated with accumulation of unmethylated CpG-rich DNA promoter fragments, implicating a mechanistic link. In a rodent model of pristane-induced lupus, administration of CpG-rich DNA (isolated from $\mathrm{LN}$ patients or synthetic unmethylated CpG-rich DNA ODN) worsened the renal phenotype. TLR9-mediated intrarenal inflammation can be therapeutically targeted by administration of synthetic methylated CpG-rich DNA oligonucleotides, ultimately associated with suppression of TLR9mediated signalling responses and renal injury in experimental LN.

Conclusions Our results implicate accumulation of unmethylated CpG-rich promoter DNA fragments in LN. Furthermore. these unmethylated $\mathrm{CpG}$-rich promoter DNA fragments causally contribute to TLR9-mediated inflammation and renal fibrogenesis and administration of methylated CpG-rich ODN attenuated intrarenal TLR9-mediated inflammatory signalling responses. Therefore, biomonitoring of $\mathrm{CpG}$-rich promoter DNA fragments and modulation of intrarenal TLR9 signalling might be a promising therapeutical target in LN.

\section{PS2:43 IS LUPUS MORE PREVALENT IN WORLD'S MOST STRESSED COUNTRIES?}

A Almathkouri, D Pyne, A Pakozdi, M Lewis, A Cove-Smith, R Rajakariar. Barts Lupus Centre, London, UK

\subsection{6/lupus-2018-abstract.91}

Background A number of studies have implicated psychological stress as a trigger for autoimmune diseases. In a questionnaire study involving 120 lupus patients emotional stress was selected in over $75 \%$ cases as a trigger for their disease. ${ }^{1}$ The role of stress as a trigger in lupus however is controversial. Here we study whether there is an association between the prevalence of lupus in various countries and their reported stress measures.

Methods We undertook a literature review of the reported prevalence of lupus in various countries across the world. We then recorded the reported stress index in those countries from Bloomberg's study, ${ }^{2}$ which utilised seven equally weighted variables: homicide rates, GDP per capita income inequality, corruption perception, unemployment, urban air pollution and life expectancy to rank 74 countries according to stress levels. Pearsons correlation was used to measure association between national stress indices and lupus prevalence

Results Results are presented in graph 1. Prevalence data was only available in the literature for limited countries. Of the countries studied no correlation was found between national stress indices and lupus prevalence $(\mathrm{r}=-0.028$, p-value 0.449). Conclusion We found no association between a country's prevalence of lupus and the measured stressfulness of its living environment. 

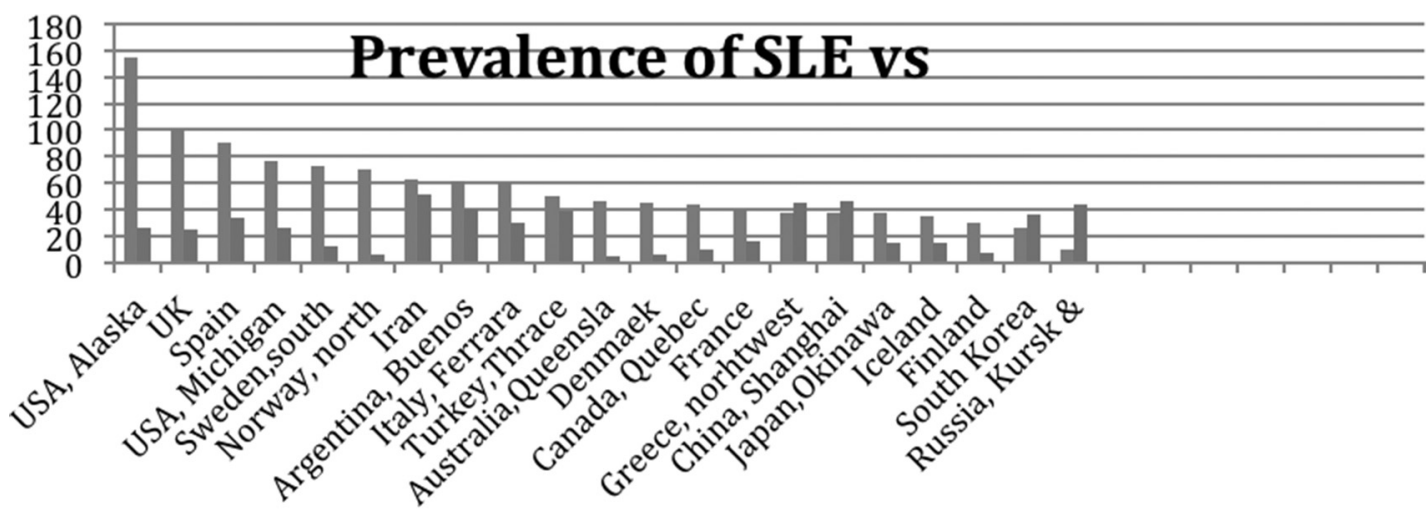

Prevalence of SLE $\quad$ stress scale

\section{Abstract PS2:43 Figure 1}

\section{REFERENCES}

1. Stojanovich L. Stress as a trigger of autoimmune disease. Abstracts book: 5 th International Congress on Autoimmunity, Sorrento, Italy, vol. 355. Autoimmun Rev; 2006.

2. World Health Organisation. United Nations Office on Drugs and Crime, International Monetary Fund, Central Intelligence Agency World Factbook, Transparency International May 2013.

\section{PS2:44 ROLE OF ANTI-DFS70 ANTIBODIES IN THE SEROLOGICAL DIAGNOSTICS OF SLE}

${ }^{1} \mathrm{~N}$ Röber, ${ }^{1} \mathrm{M}$ Achleitner, ${ }^{2} \mathrm{M}$ Aringer, ${ }^{3} \mathrm{~S}$ Rudolph, ${ }^{4} \mathrm{~L}$ Unger, ${ }^{5} \mathrm{~A}$ Gräßler, ${ }^{6} \mathrm{~K}$ Lüthke, ${ }^{7} \mathrm{M}$ Mahler, ${ }^{1} \mathrm{~K}$ Conrad. ${ }^{1}$ Institute of Immunology, Technical University Dresden, Dresden, Germany; ${ }^{2}$ Department of Medicine III, University Hospital Carl Gustav Carus, Dresden, Germany; ${ }^{3}$ Immune Centre Chemnitz, Chemnitz, Germany; ${ }^{4}$ Department of Medicine I, Municipal Hospital, Dresden-Friedrichstadt, Germany; ${ }^{5}$ Medical Practice, Pirna, Germany; ${ }^{6}$ Medical Practice of Rheumatology, Dresden, Germany; ${ }^{7}$ Inova Diagnostics, San Diego, USA

\subsection{6/lupus-2018-abstract.92}

Background Positive antinuclear antibosies (ANA) may lead to additional testing and potentially even inappropriate treatment in patients with rheumatic symptoms not caused by systemic lupus erythematosus (SLE). The aim of our study was to evaluate if autoantibodies directed against DFS70 can be used to exclude SLE in ANA positive patients.

Patients and methods Anti-DFS70 antibodies were determined by chemoluminescence assay (CIA) in sera of 352 apparently healthy individuals (AHI), 300 patients with SLE, 335 patients with other connective tissue diseases (CTD) including 56 patients with undifferentiated connective tissue disease (UCTD), and 660 non-CTD patients (302 rheumatoid arthritis, 94 ANCA-associated vasculitis, 87 atopic rhinitis, 135 paediatric patients with celiac disease, and 42 autoimmune liver diseases). Furthermore, 1048 patients of a routine cohort with positive ANA results determined by immunofluorescence on HEp-2-cells showing positive staining of the chromatine region and negative result in confirmatory assays for antibodies against dsDNA, histone, nucleosome, and DNA-topoisomerase 1 antibodies were included in this study.

Results In AHI and in the non-CTD cohort, anti-DFS70 antibodies occur with a prevalence of $5.1 \%$ and $2 \%$, respectively. Of the 1048 selected routine sera, 205 (19.6\%) were positive for anti-DFS70 antibodies. Various diseases but no definite SLE were diagnosed according to available data of 116 of anti-DFS70 positive patients. Of the 300 SLE patients, only one patient was low titred positive for anti-DFS70 antibodies in addition to dsDNA, nucleosome, Ro/SS-A and La/SS-B autoantibodies. In the non-CTD group, only 6 of 579 patients $(1.2 \%)$ were positive for anti-DFS70 antibodies, all of them also show disease specific autoantibodies. In patients with UCTD, $6(10.7 \%)$ were anti-DFS70 antibody positive in the absence of disease specific autoantibodies. Up to now, no development of SLE was observed in these patients.

Conclusion If anti-DFS70 antibodies are positive in the absence of SLE specific autoantibodies, SLE can be excluded with high certainty.

\section{Poster session 3: Epidemiology}

\section{PS3:45 SPATIAL-TIME CLUSTER ANALYSIS OF SLE DISEASE ACTIVITY}

G Stojan, F Curriero, A Kvit, M Petri. Johns Hopkins University, Baltimore, USA

\subsection{6/lupus-2018-abstract.93}

Background Cluster detection is an essential tool in the public health domain with the goal of detecting anomalous clusters of disease cases. We performed a spatial-time cluster analysis of the Johns Hopkins Lupus cohort with the goal of identifying potential spatial-time clusters of SLE organ specific disease activity.

Methods 1844 patients who fulfil 4 of the 11 American College of Rheumatology classification criteria for SLE and who had recorded home addresses were included in the analysis. Cluster detection analysis in both space and time of disease activity expressed as Physician Global Estimate (PGA) was performed. The area utilised in this analysis was a 350 kilometre radial buffer around the Johns Hopkins Lupus Centre, and included all of Maryland, Delaware, and District of Columbia, as well as parts of Pennsylvania, New Jersey, Virginia, and West Virginia. This area was considered due to the high and consistent density of study participants. The data ranged from 1987 to 2017, with the spring, summer, fall, and winter seasons serving as time units for the temporal based analyses.

Results CNS, renal, and joint flares have both seasonal patterns as well as large-scale multi-year trends. CNS flares clustered between Annapolis, MD and Frederick, MD between 1987 and 2000, renal flares clustered in central Maryland and northern Virginia between 2002 and 2006 and and a joint 